\title{
Chapter 64 \\ Characterizing Fluctuations of Arterial and Cerebral Tissue Oxygenation in Preterm Neonates by Means of Data Analysis Techniques for Nonlinear Dynamical Systems
}

\author{
Stefan Kleiser, Marcin Pastewski, Tharindi Hapuarachchi, \\ Cornelia Hagmann, Jean-Claude Fauchère, Ilias Tachtsidis, Martin Wolf, \\ and Felix Scholkmann
}

\begin{abstract}
The cerebral autoregulatory state as well as fluctuations in arterial $\left(\mathrm{SpO}_{2}\right)$ and cerebral tissue oxygen saturation $\left(\mathrm{StO}_{2}\right)$ are potentially new relevant clinical parameters in preterm neonates. The aim of the present study was to test the investigative capabilities of data analysis techniques for nonlinear dynamical systems, looking at fluctuations and their interdependence. $\mathrm{StO}_{2}, \mathrm{SpO}_{2}$ and the heart rate (HR) were measured on four preterm neonates for several hours. The fractional tissue oxygenation extraction (FTOE) was calculated. To characterize the fluctuations in $\mathrm{StO}_{2}, \mathrm{SpO}_{2}$, FTOE and HR, two methods were employed: (1) phase-space modeling and application of the recurrence quantification analysis (RQA), and (2) maximum entropy spectral analysis (MESA). The correlation between $\mathrm{StO}_{2}$ and $\mathrm{SpO}_{2}$ as well as FTOE and HR was quantified by (1) nonparametric nonlinear regression based on the alternating conditional expectation (ACE) algorithm, and (2) the maximal information-based nonparametric exploration (MINE) technique. We found that (1) each neonate showed individual characteristics, (2) a $\sim 60$ min oscillation was observed in all of the signals, (3) the nonlinear correlation strength between $\mathrm{StO}_{2}$ and $\mathrm{SpO}_{2}$ as well as FTOE and HR was specific for each neonate and showed a high value for a neonate with a reduced health status, possibly indicating an impaired cerebral
\end{abstract}

\footnotetext{
This chapter was originally published under a CC BY-NC 4.0 license, but has now been made available under a CC BY 4.0 license. An erratum to this chapter can be found at DOI 10.1007/978-1-4939-3023-4_66.

\section{S. Kleiser • M. Pastewski • M. Wolf • F. Scholkmann $(\square)$}

Biomedical Optics Research Laboratory, Department of Neonatology, University Hospital

Zurich, University of Zurich, 8091 Zurich, Switzerland

e-mail: Felix.Scholkmann@usz.ch

T. Hapuarachchi $\bullet$ I. Tachtsidis

Department of Medical Physics and Bioengineering, University College London, London, UK

C. Hagmann • J.-C. Fauchère

Division of Neonatology, University of Zurich, 8091 Zurich, Switzerland
} 
autoregulation. In conclusion, our data analysis framework enabled novel insights into the characteristics of hemodynamic and oxygenation changes in preterm infants. To the best of our knowledge, this is the first application of RQA, MESA, ACE and MINE to human $\mathrm{StO}_{2}$ data measured with near-infrared spectroscopy (NIRS).

Keywords Long term measurements - Autoregulation - Near infrared spectroscopy $\bullet$ Correlation analysis $\bullet$ Spontaneous fluctuations

\section{Introduction}

Preterm infants exhibit an immature regulation of respiration as well as systemic and cerebral blood circulation (i.e. cerebral autoregulation, $\mathrm{CO}_{2}$ vasoreactivity), leading to an increased incidence of hypoxic and hyperoxic episodes due to (1) large fluctuations in cerebral hemodynamics, and (2) impaired coupling between cerebral blood flow (CBF) and metabolic demand [1]. Episodes of intermittent hypoxemia occur in $74 \%$ of preterm infants, compared to $62 \%$ of term infants [2]. Hyperoxemia or hypoxemia may lead to an increase in mortality and neurological morbidity with long-term effects in later adult life. Greater variability in arterial oxygen saturation $\left(\mathrm{SpO}_{2}\right)$ [3] correlates with an increased incidence of retinopathy of prematurity (ROP). Thus, the assessment of the dynamics of $\mathrm{SpO}_{2}$ and cerebral tissue oxygen saturation $\left(\mathrm{StO}_{2}\right)$ in preterm neonates may be of high clinical relevance. Due to continuous advancement in biomedical optics [4, 5], a reliable noninvasive longterm measurement of $\mathrm{StO}_{2}$ in preterm neonates is in principle feasible [6, 7].

The aim of the present study was to analyze long-term measurements of $\mathrm{StO}_{2}$ (conducted by multi-distance near-infrared spectroscopy, MD-NIRS) and $\mathrm{SpO}_{2}$, heart rate (HR) and the fractional tissue extraction (FTOE) in preterm infants by means of data analysis techniques for nonlinear dynamical systems in order to investigate the characteristics of cerebral and systemic hemodynamic fluctuations and their interdependence.

\section{Material and Methods}

\subsection{Subjects, Instrumentation and Experimental Protocol}

A total of 20 clinically stable preterm neonates were enrolled. The study was approved by the ethics committee, and written informed consent was obtained from the parents before the study. Four neonates were selected for the present analysis, namely those with long continuous signals and the highest signal-to-noise ratio (SNR) (Table 64.1). $\mathrm{SpO}_{2}$ and $\mathrm{HR}$ were determined by a standard patient monitor (Infinity Delta XL, Dräger, Germany) and $\mathrm{StO}_{2}$ by an internally developed MD-NIRS device (OxyPrem, $4 \times 3$ [760, 805, $870 \mathrm{~nm}$ ] light sources, two sourcedetector distances, i.e. $1.5,2.5 \mathrm{~cm}[8])$. OxyPrem uses the self-calibrating approach 
Table 64.1 Description of the study sample

\begin{tabular}{l|l|l|l|l}
\hline Characteristics & Neonate \#1 & Neonate \#2 & Neonate \#3 & Neonate \#4 \\
\hline GA at birth (weeks) & 33.4 & 26.4 & 29.4 & 26.8 \\
\hline GA at measurement (weeks) & 34.7 & 28.5 & 29.9 & 30.7 \\
\hline Weight at measurement $(\mathrm{g})$ & 2220 & 1280 & 1090 & 1440 \\
\hline Apgar $(1,5,10)$ & $8,8,9$ & $5,4,5$ & $8,8,8$ & $5,8,8$ \\
\hline Respiration & Spontaneous & SIMV & CPAP & Spontaneous \\
\hline $\mathrm{FiO}_{2}(\%), \mathrm{Hct}(\%), \mathrm{Hb}(\mathrm{g} / \mathrm{dL})$ & $21,50.6,16.6$ & $25,40.9,13.4$ & $21,49.5,16.1$ & $21,36,11.7$ \\
\hline PDA & No & No & Yes & No \\
\hline Length of analyzed data (min) & 111 & 271 & 145 & 308 \\
\hline
\end{tabular}

GA gestational age, $\mathrm{FiO}_{2}$ fraction of inspired oxygen, $\mathrm{SIMV}$ synchronized intermittent mandatory ventilation, $C P A P$ continuous positive airway pressure, $H c t$ hematocrit, $H b$ hemoglobin, PDA persistent ductus arteriosus

[9] which ensures a robust and high-precision measurement of absolute $\mathrm{StO}_{2}$ values [10]. The NIRS optode was positioned over the left prefrontal cortex (PFC).

Measurements were performed continuously during the night (from $\sim 10 \mathrm{pm}$ till $\sim 6 \mathrm{am})$, i.e. NIRS measured the resting-state activity of cerebral hemodynamics.

\subsection{Signal Processing and Data Analysis}

From the $\mathrm{SpO}_{2}$ and $\mathrm{StO}_{2}$ we calculated the fractional tissue oxygenation extraction $\left(\mathrm{FTOE}=\left(\mathrm{SpO}_{2}-\mathrm{StO}_{2}\right) / \mathrm{SpO}_{2}\right) \times 100[\%]$. FTOE quantifies the balance between oxygen delivery and oxygen consumption and correlates significantly with the invasively measured oxygen extraction fraction [11]. All signals $\left(\mathrm{SpO}_{2}, \mathrm{StO}_{2}\right.$, FTOE and HR) were downsampled to $0.05 \mathrm{~Hz}$ to increase the SNR and since only low frequencies were of interest. For each of the four datasets, an interval was chosen for the subsequent analysis which contains data without any signal distortion. The lengths of the data are given in Table 64.1. To characterize the fluctuations in $\mathrm{StO}_{2}, \mathrm{SpO}_{2}, \mathrm{FTOE}$ and $\mathrm{HR}$, two different methods were applied:

- Phase-space modeling and application of the recurrence quantification analysis $(R Q A)[12,13]$. Each signal $\left(\mathrm{StO}_{2}, \mathrm{SpO}_{2}, \mathrm{FTOE}\right.$ and $\left.\mathrm{HR}\right)$ was embedded into a phase space with the dimension $m$ and time delay $\tau$. The optimal values for $m$ and $\tau$ were determined by finding the first minimum of the false nearest neighbors function depending on $m$, and the autocorrelation function depending on $\tau$, respectively. In a subsequent step, the phase space trajectories were characterized by the RQA. In particular, the determinism (DET, i.e. the predictability of the system), entropy of the diagonal length (ENT, i.e. the complexity of the system's deterministic dynamics), and laminarity (LAM, i.e. the amount of intermittency of the system's dynamics) were calculated.

- Maximum entropy spectral analysis (MESA) [14]. This method enables a highprecision spectral analysis based on the principle of maximum entropy. 
To prevent spurious peaks, the order of the MESA-based periodogram was set at one third of the number of samples [15].

The correlation between $\mathrm{StO}_{2}$ and $\mathrm{SpO}_{2}$ as well as FTOE and HR were quantified by two nonparametric methods:

- Nonparametric nonlinear regression based on the alternating conditional expectation (ACE) algorithm [16]. This technique finds the optimal transformations for the dependent and independent variables in order to maximize the correlation. The correlation strength is quantified by the maximal correlation coefficient, $r_{\mathrm{ACE}}$.

- Maximal information-based nonparametric exploration (MINE) technique [17]. MINE enables the characterization of dependencies between variables. We calculated the maximal information coefficient (MIC) (relationship strength) and maximum asymmetry score (MAS) (departure from monotonicity).

In addition, each signal was characterized by calculating the median, and variability index $1\left(V I_{1}\right.$, quantified as the mean of the modulus of the first derivation). In addition, the relationship of the fluctuation strength of $\mathrm{StO}_{2}$ vs. $\mathrm{SpO}_{2}$ was determined by the ratio of their standard deviations (variability index $2, V I_{2}$ ).

\section{Results, Discussion, Conclusion and Outlook}

Figure 64.1a-d shows the time courses of $\mathrm{SpO}_{2}, \mathrm{StO}_{2}, \mathrm{HR}$ and FTOE. In Fig. 64.1b the normalized (i.e. subtraction of the mean value) $\mathrm{SpO}_{2}$ and $\mathrm{StO}_{2}$ were plotted to increase the visibility of similar dynamics. Figure 64.1d shows FTOE and HR after normalization (z-score) and smoothing (Kolmogorov-Zurbenko filter, window length: $180 \mathrm{~s}$, iterations: 2) which increases the visibility of the similar long-term variability of both signals. The ACE correlation plots as well as the RQA, ACE and MINE results are visualized in Fig. 64.2. All signals show subject-specific dynamics:

- RQA: Noticeable low values for DET and LAM in neonate \#4, high values for neonate \#1. ENT (for $\mathrm{StO}_{2}$ and $\mathrm{SpO}_{2}$ ) has low values for neonate \#3.

- MESA: (1) Neonate \#1 exhibited a large oscillation with a period length $(T)$ of 60 min in $\mathrm{StO}_{2}$ and $\mathrm{SpO}_{2}$ as well as HR and FTOE. (2) A large oscillation with $T \approx 30 \mathrm{~min}$ is present in neonate \#2 for $\mathrm{StO}_{2}$ and $\mathrm{SpO}_{2}$, followed by a second strongest oscillation with $T \approx 60 \mathrm{~min}$. In FTOE the predominant oscillation was at $T \approx 60$ min. The spectra of $\mathrm{StO}_{2}$ and $\mathrm{SpO}_{2}$ have a remarkably similar fine structure of oscillatory peaks indicating a large degree of similarity in the dynamics. In addition, an oscillation with $T \approx 15 \min$ can be seen in $\mathrm{StO}_{2}$, $\mathrm{SpO}_{2}$, HR and FTOE. (3) Neonate \#3 shows an oscillation peak with $T \approx 60$ min in $\mathrm{StO}_{2}, \mathrm{SpO}_{2}, \mathrm{HR}$ and $\mathrm{FTOE}$, whereas for FTOE a larger oscillation with $T \approx 30$ min is present. The spectra of $\mathrm{StO}_{2}$ and $\mathrm{SpO}_{2}$ have different fine 


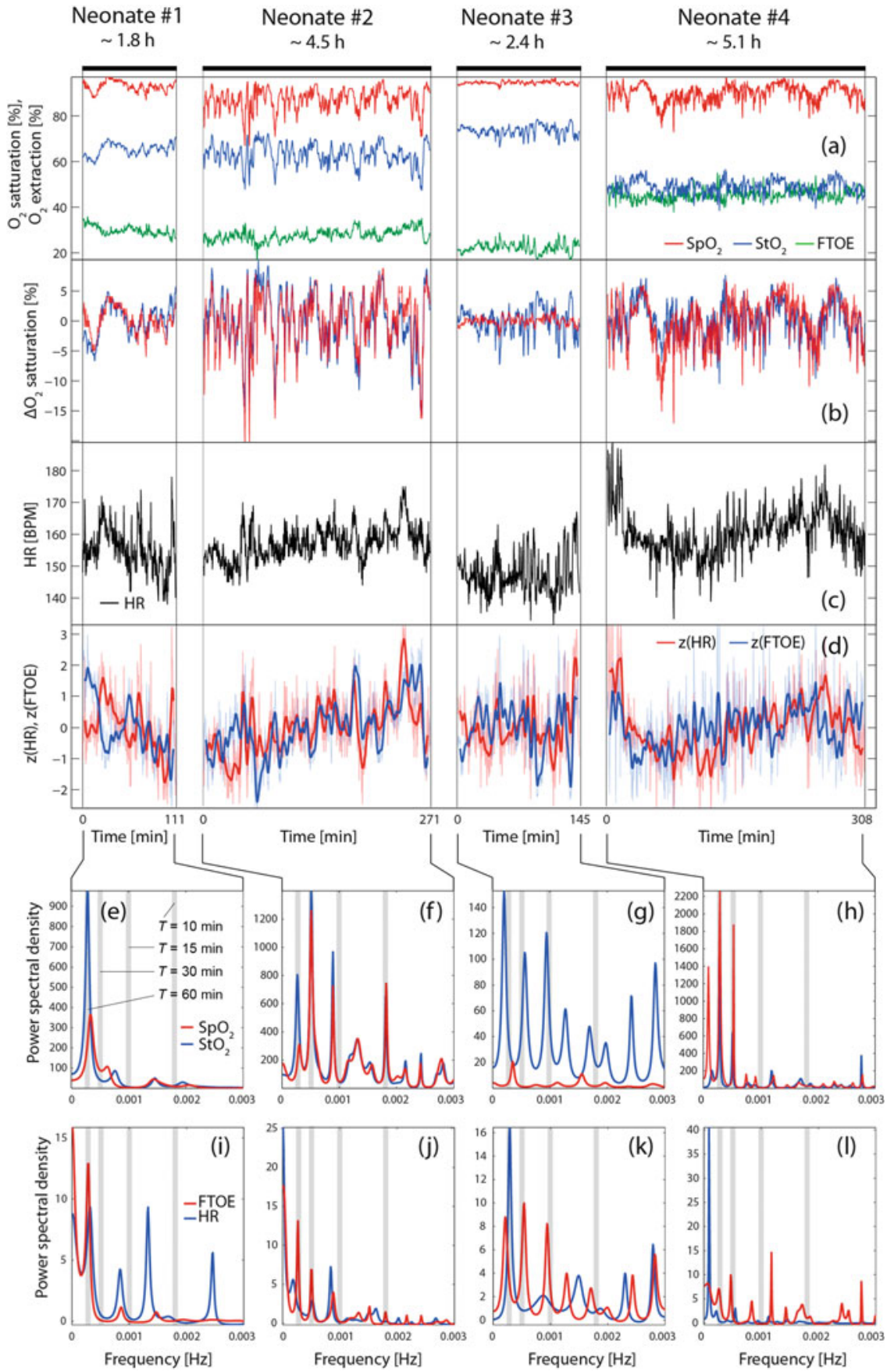

Fig. 64.1 (a-d) Visualization of the analyzed signals $\left(\mathrm{StO}_{2}, \mathrm{SpO}_{2}\right.$, FTOE and HR). (e-l) Frequency spectra obtained by MESA 
(a)

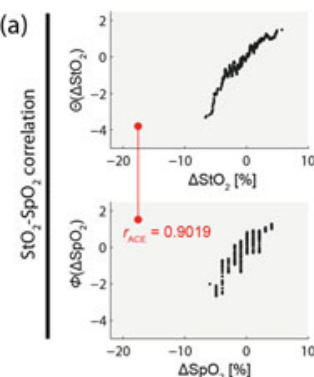

(b)

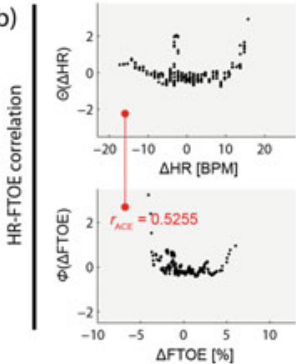

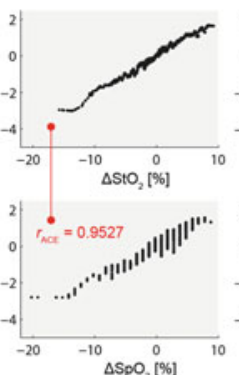
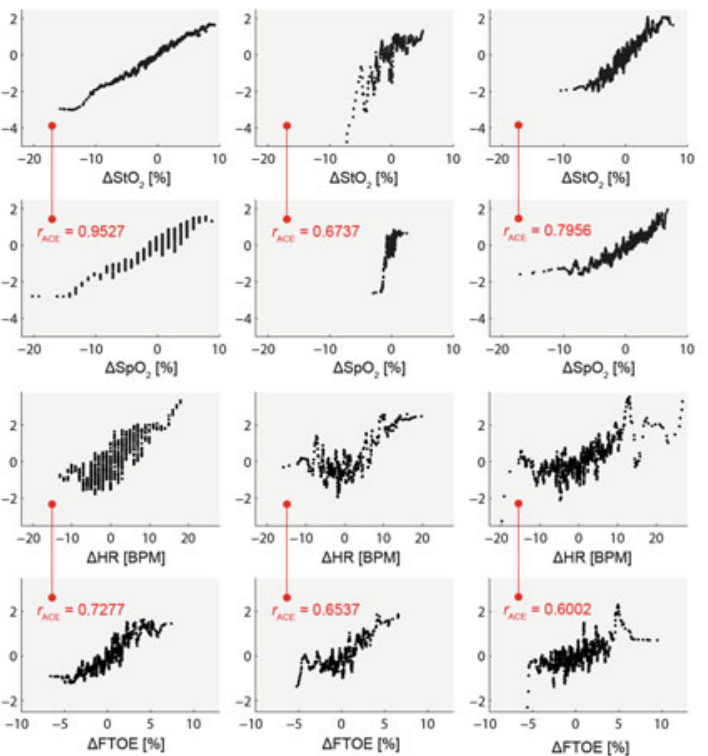

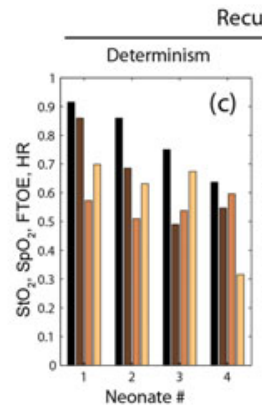

Recurrence quantification analyis (RQA)
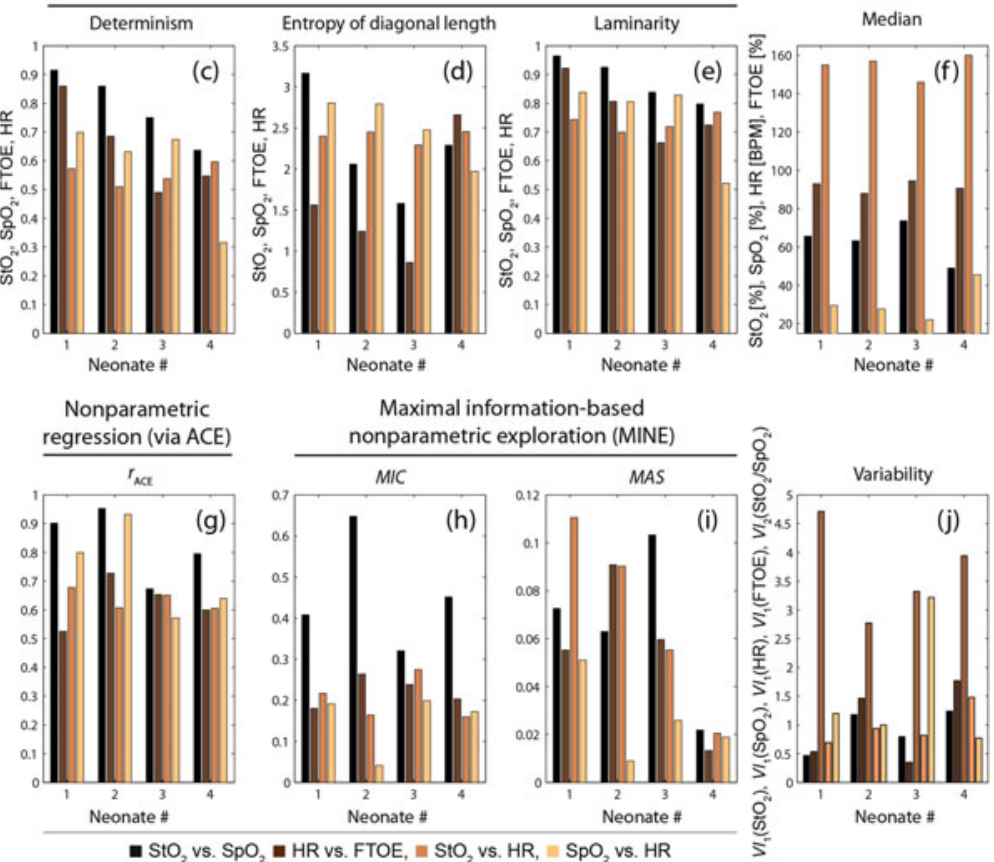

Fig. 64.2 (a, b) Correlation diagrams based on ACE nonparametric nonlinear regression. $(\mathbf{c}-\mathbf{j})$ Parameters obtained by RQA, MESA, ACE, MINE as well as the values for the median and variability 
structures, which is also true for the spectra of HR and FTOE. (4) Neonate \#4 shows a strong peak with $T \approx 60$ and 30 min in $\mathrm{StO}_{2}$ and $\mathrm{SpO}_{2}$.

- ACE and MINE: Concerning the relationship between $\mathrm{StO}_{2}$ and $\mathrm{SpO}_{2}$, the largest $r_{\mathrm{ACE}}$ and $M I C$ value was found for neonate \#2, the lowest for neonate \#3; MAS was highest in neonate \#3. Concerning HR vs. FTOE, neonate \#2 had the largest $r_{A C E}, M I C$ and $M A S$ values. Neonate \#4 showed significantly low $M A S$ values for all four conditions $\left(\mathrm{StO}_{2}\right.$ vs. $\mathrm{SpO}_{2}$ or $\mathrm{HR}, \mathrm{HR}$ vs. FTOE or $\mathrm{SpO}_{2}$ ).

To interpret the results it is helpful to discuss the similarities and differences of the signal characteristics with respects to the four neonates:

- Similarities: (1) The values for DET, ENT and LAM were all higher for $\mathrm{StO}_{2}$ compared to $\mathrm{SpO}_{2}$ (except for ENT of neonate \#4), indicating more complex signal characteristics of $\mathrm{StO}_{2}$ than of $\mathrm{SpO}_{2}$. (2) The correlation between $\mathrm{StO}_{2}$ and $\mathrm{SpO}_{2}$ is higher than that observed between HR and FTOE $\left(r_{\mathrm{ACE}}\left(\mathrm{StO}_{2}\right.\right.$, $\left.\mathrm{SpO}_{2}\right)=0.8310 \pm 0.1236, \quad M I C\left(\mathrm{StO}_{2}, \quad \mathrm{SpO}_{2}\right)=0.4570 \pm 0.1385 ; \quad r_{\mathrm{ACE}}(\mathrm{HR}$, $\mathrm{FTOE})=0.6268 \pm 0.0854, \operatorname{MIC}(\mathrm{HR}, \mathrm{FTOE})=0.2216 \pm 0.0371)$. (3) All neonates showed an oscillation in $\mathrm{StO}_{2}, \mathrm{SpO}_{2}, \mathrm{FTOE}$ and $\mathrm{HR}$ with a period of $T \approx 60 \mathrm{~min}$, whereas the amplitude was specific for each neonate.

- Differences: (1) DET, LAM and ENT of $\mathrm{SpO}_{2}$ were highest for neonate \#3. (2) The correlation $\left(r_{\mathrm{ACE}}, M I C\right)$ between $\mathrm{StO}_{2}$ and $\mathrm{SpO}_{2}$ as well as $\mathrm{HR}$ and FTOE was highest for neonate \#2; neonate \#3 showed the lowest $\mathrm{StO}_{2} / \mathrm{SpO}_{2}$ correlation. (3) The smallest $M A S$ values for all four correlations were found for neonate \#4 (except for $\mathrm{StO}_{2}$ vs. HR, neonate \#2). (4) In neonate \#3 we observed the highest median values for $\mathrm{StO}_{2}$ and $\mathrm{SpO}_{2}$ as well as the lowest ones for HR and FTOE. In neonate \#4 the lowest $\mathrm{StO}_{2}$ and the highest FTOE value were measured. (5) High $V I_{1}$ values in $\mathrm{StO}_{2}$ and $\mathrm{SpO}_{2}$ were present in neonates \#2 and \#4. Neonate \#3 showed the largest $V I_{2}$ value for $\mathrm{StO}_{2} / \mathrm{SpO}_{2}$. (6) The mean $\mathrm{StO}_{2}$ values correlated inversely with the individual Hct and GA (at birth) $(r=-0.927$ and $r=-0.982$, respectively; $p<0.05)$.

The physiological interpretation of these findings is not straightforward since all patient-specific characteristics have an influence on the analyzed parameters. In particular, the general health state (e.g. PDA, microbleeds, ischemia: yes/no), the type of respiration (ventilatory support: yes/no, type of support), and the GA (at birth/measurement) could potentially have a strong impact on the parameters. The following observations were made based on our analysis: (1) The general inverse correlation observed between $\mathrm{StO}_{2}$ and Hct was also noticed by other studies (e.g. [18]). (2) Neonate \#3 exhibited a large $V I_{2}$, e.g. the fluctuations in $\mathrm{StO}_{2}$ were much stronger than in $\mathrm{SpO}_{2}$ (especially the decreases), a pattern that is observed by neonates with a PDA-indeed, neonate \#3 had a PDA (which was however classified as not hemodynamically relevant). The low $\mathrm{StO}_{2} / \mathrm{SpO}_{2}$ correlation $\left(r_{\mathrm{ACE}}, M I C\right)$ and the different frequency spectra $\left(\mathrm{StO}_{2}\right.$ vs. $\mathrm{SpO}_{2}, \mathrm{HR}$ vs. FTOE) point also to a specific state of the systemic-cerebral hemodynamic coupling. The observation that neonate \#3 had the highest median values for $\mathrm{StO}_{2}$ and $\mathrm{SpO}_{2}$ as 
well as the lowest ones for HR and FTOE is surprising since one would expect an increased FTOE and decrease $\mathrm{StO}_{2}$ in case of a PDA [19]. (3) The oscillations in the data with $T \approx 60$ and $30 \mathrm{~min}$ could originate from sleep phases. A sleep-wake cycling (with a quiet sleep phase with $T \approx 20 \mathrm{~min}$ ) is known [20] in term newborns with $T \approx 50-60 \mathrm{~min}$ and an increase in total hemoglobin and HR during active sleep (compared to quite sleep) has previously been observed [21, 22]. (4) The two neonates with the lowest GA at birth (\#2, \#4) had the largest variability of $\mathrm{StO}_{2}$, $\mathrm{SpO}_{2}$ and FTOE which could indicate an immature functioning of cerebral hemodynamic regulation.

In conclusion, using four case studies, we demonstrated the possibility of realizing long-term measurements in preterm neonates with MD-NIRS and we presented a novel framework for investigating the characteristics of cerebral and systemic hemodynamic fluctuations and their interdependence. A follow-up study, investigating the signal characteristics in healthy and ill preterm neonates using the same framework would be the next step. Focusing on the fluctuation characteristics of the signals may offer novel insights into systemic and cerebral hemodynamics that are not assessed routinely only using traditional analyses (i.e. based on moments and linear correlations). To the best of our knowledge, this is the first application of RQA, MESA, ACE and MINE to human NIRS data.

Acknowledgments The work was supported by the Wellcome Trust. We gratefully acknowledge funding by Nano-Tera (NeoSense, ObeSense), the Swiss National Science Foundation, the Neuroscience Center Zurich (UCL-Zurich Collaboration), The Danish Council for Strategic Resarch (SafeBoosC). We thank Caroline Guyer MD (Children's Hospital Zurich) for her valuable input, and Rachel Scholkmann for proofreading the manuscript.

\section{References}

1. Fyfe KL et al (2013) The development of cardiovascular and cerebral vascular control in preterm infants. Sleep Med Rev 18:299-310

2. Hunt $\mathrm{CE}$ et al (2011) Longitudinal assessment of hemoglobin oxygen saturation in preterm and term infants in the first six months of life. J Pediatr 159(3):377-383

3. Di Fiore JM et al (2012) The relationship between patterns of intermittent hypoxia and retinopathy of prematurity in preterm infants. Pediatr Res 72(6):606-612

4. Scholkmann F et al (2014) A review on continuous wave functional near-infrared spectroscopy and imaging instrumentation and methodology. Neuroimage 85(Part 1):6-27

5. Wolf M, Ferrari M, Quaresima V (2007) Progress of near-infrared spectroscopy and topography for brain and muscle clinical applications. J Biomed Opt 12(6):062104

6. Wolf M, Greisen G (2009) Advances in near-infrared spectroscopy to study the brain of the preterm and term neonate. Clin Perinatol 36(4):807-834

7. Wolf $\mathrm{M}$ et al (2012) A review of near infrared spectroscopy for term and preterm newborns. J Near Infrared Spectrosc 20(1):43-55

8. Hyttel-Sorensen S et al (2013) Calibration of a prototype NIRS oximeter against two commercial devices on a blood-lipid phantom. Biomed Opt Express 4(9):1662-1672

9. Hueber DM et al (1999) New optical probe designs for absolute (self-calibrating) NIR tissue hemoglobin measurements. Opt Tomogr Spectrosc Tissue III Proc SPIE 3597:618-631 
10. Scholkmann F, Metz AJ, Wolf M (2014) Measuring tissue hemodynamics and oxygenation by continuous-wave functional near-infrared spectroscopy - how robust are the different calculation methods against movement artifacts? Physiol Meas 35(4):717-734

11. Naulaers G et al (2007) Use of tissue oxygenation index and fractional tissue oxygen extraction as non-invasive parameters for cerebral oxygenation. A validation study in piglets. Neonatology $92(2): 120-126$

12. Webber CL Jr, Zbilut JP (1985) Dynamical assessment of physiological systems and states using recurrence plot strategies. J Appl Physiol 76(2):965-973

13. Marwan $\mathrm{N}$ et al (2002) Recurrence plot based measures of complexity and its application to heart rate variability data. Phys Rev E 66(2):026702

14. Ulrych TJ, Bishop TN (1975) Maximum entropy spectral analysis and autoregressive decomposition. Rev Geophys 13(1):183-200

15. Kay SM (1979) The effects of noise on the autoregressive spectral estimator. IEEE Trans Acoust Speech Signal Process 27(5):478-485

16. Breiman L, Friedman JH (1985) Estimating optimal transformations for multiple regression and correlation. J Am Stat Assoc 80(391):580-598

17. Reshef DN et al (2011) Detecting novel associations in large data sets. Science 334 (6062):1518-1524

18. Youkin DP et al (1987) The effect of hematocrit and systolic blood pressure on cerebral blood flow in newborn infants. J Cereb Blood Flow Metab 7(3):295-299

19. Vanderhaegen J et al (2008) Surgical closure of the patent ductus arteriosus and its effect on the cerebral tissue oxygenation. Acta Paediatr 97(12):1640-1644

20. Osredkar D et al (2005) Sleep-wake cycling on amplitude-integrated electroencephalography in term newborns with hypoxic-ischemic encephalopathy. Pediatrics 115(2):327-332

21. Münger DM, Bucher HU, Duc G (1998) Sleep state changes associated with cerebral blood volume changes in healthy term newborn infants. Early Hum Dev 52(1):27-42

22. Stéphan-Blanchard E et al (2013) Heart rate variability in sleeping preterm neonates exposed to cool and warm thermal conditions. PLoS One 8(7):e68211

Open Access This chapter is licensed under the terms of the Creative Commons Attribution 4.0 International License (http://creativecommons.org/licenses/by/4.0/), which permits use, sharing, adaptation, distribution and reproduction in any medium or format, as long as you give appropriate credit to the original author(s) and the source, provide a link to the Creative Commons license and indicate if changes were made.

The images or other third party material in this chapter are included in the chapter's Creative Commons license, unless indicated otherwise in a credit line to the material. If material is not included in the chapter's Creative Commons license and your intended use is not permitted by statutory regulation or exceeds the permitted use, you will need to obtain permission directly from the copyright holder.

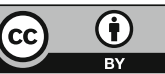

